\title{
Vegetation Database of Pine Forests on Acidic Soils in Germany
}

\author{
Thilo Heinken
}

\begin{abstract}
The Vegetation Database of Pine Forests on Acidic Soils in Germany (GIVD ID EU-DE-019) of \pm acidophytic pine forests (mainly Dicrano-Pinion and plantations at deciduous forest sites and Pulsatillo-Pinetea) in Germany comprises 3327 relevés, about two thirds from the Northern German lowlands and one third from the hilly regions up to approximately 800 m a.s.l. (mainly Bavaria). Besides Dicrano-Pinion, also small amounts of Vaccinio uliginosi-Pinetea and montain scree forest relevés (Betulo carpaticaeSorbetum) are included. The database was created in the early 1990s and finished in 2005 in order to re-evaluate the syntaxonomy of acidophytic pine forests of Germany based on \pm all available vegetation data. Only original relevés (Braun-Blanquet method) from 73 sources, published in local ecological journals or monographs, or unpublished material e.g. from diploma theses, are included. Plots sizes, complete structural data and exact georeference are mostly not included in the electronic repository, but may be retrieved from the original publications in many cases. Beyond phytosociological classification and analysis of large-scale geographic patterns within plant communities, parts of the database have been intensively used to evaluate site characteristics of plant communities, performance of indicator plants and the influence of pollution and land-use change on present-day vegetation. Hosted by the Department of Biodiversity Research and Botany of the University of Potsdam, the data are available for collaborative research.
\end{abstract}

Keywords: ecoinformatics; forest site classification.

\begin{tabular}{|c|c|}
\hline GIVD Database ID: EU-DE-019 & Last update: $2012-05-03$ \\
\hline \multicolumn{2}{|c|}{ Vegetation Database of Pine Forests on Acidic Soils in Germany } \\
\hline \multicolumn{2}{|c|}{$\begin{array}{l}\text { Scope: A database of acidophytic pine forests (mainly Dicrano-Pinion) in Germany. Only original relevés (Braun-Blanquet method; published in } \\
\text { local ecological journals or monographs, or unpublished) are included. }\end{array}$} \\
\hline Status: finished & Period: - \\
\hline \multicolumn{2}{|c|}{ Database manager(s): Thilo Heinken (heinken@uni-potsdam.de) } \\
\hline \multicolumn{2}{|c|}{ Owner: Thilo Heinken (private) } \\
\hline \multicolumn{2}{|l|}{ Web address: $[\mathrm{NA}]$} \\
\hline Availability: free upon request & Online search: no \\
\hline Database format(s): TABWIN & Export format(s): [NA] \\
\hline \multicolumn{2}{|l|}{ Publication: $[N A]$} \\
\hline Plot type(s): normal plots & Plot-size range: $50-900 \mathrm{~m}^{2}$ \\
\hline Non-overlapping plots: 3,327 & Estimate of existing plots: $4,500 \quad$ Completeness: $74 \%$ \\
\hline Total plot observations: 3,327 & Number of sources: 73 \\
\hline \multicolumn{2}{|l|}{ Countries: DE: $100.0 \%$} \\
\hline \multicolumn{2}{|c|}{ Forest: $[\mathrm{NA}]$ - Non-forest: [NA] } \\
\hline \multicolumn{2}{|c|}{ Guilds: all vascular plants: $100 \%$; bryophytes (terricolous or aquatic): 100\%; lichens (terricolous or aquatic): $100 \%$} \\
\hline \multicolumn{2}{|c|}{$\begin{array}{l}\text { Environmental data: altitude: } 32 \% \text {; slope aspect: } 41 \% \text {; slope inclination: } 37 \% \text {; soil depth: } 3 \% \text {; surface cover other than plants (open soil, litter, } \\
\text { bare rock etc.): } 10 \% \text {; soil pH: } 11 \% \text {; other soil attributes: } 5 \%\end{array}$} \\
\hline \multicolumn{2}{|c|}{ Performance measure(s): cover: $100 \%$} \\
\hline \multicolumn{2}{|c|}{$\begin{array}{l}\text { Geographic localisation: point coordinates less precise than GPS, up to } 1 \mathrm{~km}: 10 \% \text {; small grid (not coarser than } 10 \mathrm{~km}): 10 \% \text {; political units or } \\
\text { only on a coarser scale }(>10 \mathrm{~km}): 100 \%\end{array}$} \\
\hline $\begin{array}{l}\text { Sampling periods: } 1930-1939: \\
3.0 \%\end{array}$ & 59: 8.0\%; 1960-1969: 21.0\%; 1970-1979: 13.0\%; 1980-1989: 4.0\%; 1990-1999: 50.0\%; 2000-2009: \\
\hline
\end{tabular}

Thilo Heinken (heinken@uni-potsdam.de)

Biodiversity Research / Botany, University of Potsdam, 14471 Potsdam, GERMANY 